\title{
Atenção Primária à Saúde para atendimento às famílias de crianças com necessidades especiais
}

\author{
Primary Health Care to meet families of children with special needs
}

\section{Como citar este artigo:}

Schuertz AL, Mazza VA, Seidel B, Ruthes VBTNM, Macedo LC. Primary Health Care to meet families of children with special needs. Rev Rene. 2020;21:e43369. DOI: https://doi.org/10.15253/2175-6783.20202143369

(D)Amanda Louyse Schuertz
(D) Verônica de Azevedo Mazza ${ }^{1}$
(D) Bruna Seidel ${ }^{1}$
(D) Victoria Beatriz Trevisan Nobrega Martins Ruthes ${ }^{1}$
(D) Laura Christina Macedo ${ }^{1}$

${ }^{1}$ Universidade Federal do Paraná.

Curitiba, PR, Brasil.

\author{
Autor correspondente: \\ Amanda Louyse Schuertz \\ Rua Doutor Faivre, 1192, Apto. 401 \\ Centro, CEP: 80060-140. \\ Curitiba, PR, Brasil. \\ E-mail: amandaloouyse@gmail.com
}

\begin{abstract}
RESUMO
Objetivo: compreender a organização do serviço de Atenção Primária à Saúde para atendimento às famílias de crianças com necessidades especiais. Métodos: pesquisa qualitativa, desenvolvida com 33 profissionais, mediante entrevista semiestruturada. Utilizou-se da análise categorial temática, com auxílio do software WebQDA®. Resultados: emergiram duas categorias: Potencialidades e fragilidades na organização do atendimento às crianças com necessidades especiais, a qual evidenciou ambiguidade na assistência em relação à compreensão dos direitos e participação das famílias no acompanhamento; e Infraestrutura dos serviços para atendimento às crianças com necessidades especiais, na qual foram expressas limitações na organização dos serviços e nas Redes de Atenção à Saúde. Conclusão: notou-se ambiguidade e despreparo dos serviços em relação ao atendimento dessas crianças, na qualificação da equipe, bem como na exiguidade de protocolos específicos para normatizar tal atendimento.
\end{abstract}

Descritores: Crianças com Deficiência; Atenção Primária à Saúde; Serviços de Saúde da Criança; Família.

\begin{abstract}
Objective: to understand the organization of the Primary Health Care service to meet families of children with special needs. Methods: qualitative research, developed with 33 professionals, through semi-structured interviews. We used thematic categorical analysis, with the aid of the WebQDA® software. Results: we raised two categories: Strengths and weaknesses in the organization of the care of children with special needs, which showed ambiguity in the assistance in relation to the understanding of the rights and participation of families in the follow-up; and Infrastructure of services for meeting children with special needs, where limitations were expressed in the organization of services and in Health Care Networks. Conclusion: we perceived ambiguity and unpreparedness of services in relation to the care of these children, in the qualification of the team, as well as the lack of specific protocols to standardize this assistance.

Descriptors: Disabled Children; Primary Health Care; Child Health Services; Family.
\end{abstract}




\section{Introdução}

Estabelecida como modelo de proteção à saúde, a Constituição Federal reconhece que a saúde é direito de todos e dever do Estado, sendo os serviços de Atenção Primária à Saúde principal porta de entrada para o Sistema Único de Saúde, fundamentado na cobertura universal e oferta de serviços integrais, organizados a partir das Redes de Atenção à Saúde ${ }^{(1)}$, as quais favorecem as articulações entre os serviços, potencializando o cuidado integral ${ }^{(2)}$.

Os princípios do Sistema Único de Saúde, de integralidade e equidade, aparecem também como diretrizes norteadoras da Rede de Cuidados à Pessoa com Deficiência, com inclusão de ações na Atenção Primária à Saúde, desenvolvidas por profissionais que conhecem a dinâmica e o dia a dia da comunidade ${ }^{(3)}$.

Pessoas com deficiências são aquelas que têm incapacidades em longo prazo, de natureza física, mental, intelectual ou sensorial. São indivíduos que entram em contato com diversos obstáculos que podem ocasionar a interrupção da participação integral e completa destes na sociedade, em igualdade de condições com as demais ${ }^{(4-5)}$. Entre essas pessoas, têm-se as crianças com necessidades especiais, aquelas com deficiências e problemas crônicos de desenvolvimento físico e ou de comportamento, que causam impacto na qualidade de vida ${ }^{(6)}$. De acordo com a Pesquisa Nacional de Saúde da Criança, em 2016, 19,4\% das crianças e jovens tinham necessidades especiais de saúde ${ }^{(7)}$.

Crianças com necessidades especiais têm necessidades gerais de saúde como as demais, porém, com maior intensidade e frequência, podendo ser também mais diversas e extensas. Portanto, precisam de acesso aos serviços de qualidade que atendam às necessidades dessas, bem como das respectivas famílias, e que as apoiem nas transições para novos e diferentes serviços. 0 serviço de cuidado domiciliar tem sido associado à redução da internação hospitalar dessas crianças por condições crônicas complexas. Para obter resultados positivos, as crianças com ne- cessidades especiais precisam de redes fortes e positivas de apoio, incluindo famílias, prestadores de cuidados de saúde, entre outros serviços ${ }^{(8)}$.

Para garantir a capacidade das famílias de cuidar de uma criança com necessidades especiais, é importante que estejam disponíveis suportes que atendam às necessidades de saúde desta, uma vez que a família é a principal fonte de força e apoio. Para atender às famílias, os profissionais necessitam de conhecimento para subsidiá-las no manejo dessas crianças, para tal, é necessário observar a interação entre a saúde da criança e a estrutura familiar, pois essas famílias apresentam alto nível de estresse, sendo o suporte dos profissionais da Atenção Primária à Saúde impactante na capacidade de cuidar dessas famílias ${ }^{(8)}$.

É importante que as instituições de saúde, educação e a comunidade desenvolvam aliança entre a família e os serviços, com objetivo comum para os melhores interesses das crianças com necessidades especiais, para tanto, precisam considerar as necessidades, os pontos fortes e fracos dessas crianças e das famílias. Essas atividades podem ter impactos extensos e profundos nos aspectos físico, social, financeiro e emocional das famílias ${ }^{(8)}$, os serviços de saúde são desafiados a apoiar e a acompanhar as famílias no atendimento a essas $\operatorname{crianças}^{(9)}$.

A experiência no atendimento às crianças com necessidades especiais na residência multiprofissional em Estratégia Saúde da Família instigou o interesse pelo tema abordado. Justifica-se a pesquisa por considerar indispensável averiguar como a organização da Estratégia Saúde da Família pode proporcionar fonte usual de cuidados, importante para otimizar a atenção, o funcionamento e a qualidade de vida dessas pessoas e os desafios futuros de vidas, uma vez que o acesso à Atenção Primária à Saúde pode garantir bons resultados de saúde para essas crianças ${ }^{(8)}$. Para permitir que a comunidade usufrua integralmente do Sistema Único de Saúde, são primordiais o empoderamento da população e a qualidade do relacionamento da população com os serviços ${ }^{(7)}$, além de acompanhamento longitudinal e com responsabilidade para fortaleci- 
mento da coordenação do cuidado ${ }^{(3)}$.

Diante do exposto, questionou-se: como está organizado o serviço de saúde para atendimento às crianças com necessidades especiais, na visão dos profissionais da Atenção Primária à Saúde. Assim, objetivou-se compreender a organização do serviço de Atenção Primária à Saúde para o atendimento às famílias de crianças com necessidades especiais.

\section{Métodos}

Pesquisa qualitativa, cuja coleta de dados ocorreu durante o primeiro semestre de 2019, em município da região metropolitana de Curitiba, PR, Brasil. A organização dos serviços de saúde do município está subdividida em três distritos sanitários, com total de 25 Unidades de Saúde, destas, 18 estão organizadas pela Estratégia Saúde da Família e sete, pela atenção básica.

Para seleção dos participantes, sortearam-se duas Unidades de Saúde com Estratégia Saúde da Família, por Distrito Sanitário, para se obter representatividade do contexto do município. Elegeu-se um representante de cada categoria profissional da equipe Estratégia Saúde da Família por Unidade de Saúde: Agente Comunitário de Saúde, Auxiliar de Saúde Bucal, Cirurgião Dentista, Enfermeiro, Médico e Técnico de Enfermagem. Optou-se por entrevistar todos os profissionais que compunham a equipe de saúde, pois são percebidos como protagonistas na atenção à saúde de crianças com necessidades especiais.

Os dados foram coletados por meio de entrevista semiestruturada, com 33 profissionais de saúde de seis Unidades de Saúde da Estratégia Saúde da Família do município, com duração aproximadamente de dez minutos. 0 roteiro de entrevista abordou: dados dos participantes, organização do atendimento nas Unidades de Saúde e direitos das crianças com necessidades especiais. A fim de abranger os fenômenos estudados, utilizou-se da pergunta norteadora: quais as facilidades e dificuldades no atendimento a crianças com necessidades especiais? As questões complemen- tares foram: como as crianças com necessidades especiais são atendidas neste serviço? Como acontece a referência e contrarreferência do atendimento a essas crianças?

As entrevistas foram registradas em áudio, por meio de gravador digital, transcritas em documento no formato Word ${ }^{\circledR}$ e analisadas na íntegra, pela técnica de análise categorial temática ${ }^{(10)}$. A técnica analítica utilizada permite desmembrar os discursos coletados em categorias temáticas, de forma eficaz, a fim de identificar as respostas para o objeto do estudo, permitindo, assim, ao pesquisador inferir e interpretar os $\operatorname{dados}^{(10)}$.

A análise dos dados permitiu agrupar os resultados em duas categorias temáticas, que foram exemplificadas com trechos significativos e organizados no software WebQDA®, versão 2017. Os entrevistados foram representados por letras, conforme a categoria profissional: Auxiliar de Saúde Bucal (AB), Agente Comunitário de Saúde (AC), Cirurgião Dentista (D), Enfermeiro (E), Médico (M) e Técnico de Enfermagem (T), e numeral, por ordem sequencial da realização das entrevistas, para preservar anonimato e sigilo.

Este estudo foi aprovado pelo Comitê de Ética em Pesquisa da Universidade Federal do Paraná, do Setor de Ciências da Saúde, conforme parecer no 2.327.633/2017.

\section{Resultados}

Participaram da pesquisa seis agentes comunitários de saúde, cinco auxiliares de saúde bucal, cinco cirurgiões dentistas, seis enfermeiros, cinco medicos e seis tecnicos de enfermagem. Os participantes tinham, em média, 40 anos de idade. Em uma das unidades, não houve participação dos profissionais de saúde bucal, pois não havia serviço de odontologia; e, em outra, não havia o profissional médico, no período da coleta de dados.

As categorias temáticas que emergiram das falas foram: Potencialidades e fragilidades na organização do atendimento às crianças com necessidades 
especiais; e Infraestrutura dos serviços para atendimento às crianças com necessidades especiais.

\section{Potencialidades e fragilidades na organização do atendimento às crianças com necessidades espe- ciais}

Em relação ao atendimento às crianças com necessidades especiais, os entrevistados apontaram potencialidades e fragilidades, conforme as falas: Quando se faz o atendimento da criança, você acaba abrangendo a criança e a família (D3). Eu vejo a satisfação em poder ajudar, adoro auxiliar, adoro fazer meu trabalho, gosto muito de criança (AB4). Trabalhar com criança já é difícil, ela sendo portadora de alguma deficiência é bem mais (T2).

Os entrevistados evidenciaram ambiguidade no atendimento às crianças com necessidades especiais, tanto em relação à compreensão dos direitos dessas crianças, quanto na interpretação dos princípios de igualdade e equidade no Sistema Único de Saúde, conforme demonstram as falas: Direito à família, à moradia, direito à atenção, respeito, tem que ter isso, independente se seja da família ou da sociedade (D6). Elas têm prioridades nos atendimentos tanto na unidade quanto em qualquer outro lugar (AC3). A gente não vai deslocar uma criança que tem dificuldade de locomoção para vir até aqui desnecessariamente (M4). Tenta ter uma sensibilidade maior, porém, acaba que é uma consulta como qualquer outra (M3). Prioridade, chegar e aguardar, passar na frente, não. Não existe (T2).

Referente à adesão e participação da família no acompanhamento das crianças com necessidades especiais, os profissionais relataram que: Normalmente, mães que têm criança especial, elas já sabem tudo (E4). A família tem que participar de tudo, ela tem que saber como fazer, como agir e orientar a criança ou a pessoa que tenha problema (AB2). Essas crianças com deficiências, elas não vêm até nós, nós que temos que ir atrás delas (AC6).

\section{Infraestrutura dos serviços para atendimento às crianças com necessidades especiais}

Apesar de relatarem o esforço para atender às crianças com necessidades especiais da melhor ma- neira possível, os entrevistados reconheceram limitações na organização dos serviços, como ausência de protocolos específicos, necessidade de capacitação dos profissionais, ambiência pouco preparada para atender a esse público, escassez de materiais e insumos disponíveis. Eu acho que a gente não tem assim uma certa orientação do que fazer quando chega uma criança com deficiência (M6). A gente, como profissional, tenta dar o melhor, tenta dar uma atenção maior (M3). A gente teve algumas coisas durante a faculdade, mas nada assim específico do município voltado para o trabalho. Acho que seria até legal se tivesse (M5). Aqui na unidade de saúde, a gente não tem estrutura e nem material para atender à criança com deficiência (D1). Nós não temos rampas de acesso, a rua é horrível, não é só a da unidade, é de toda a localização da nossa área de abrangência, não é local de fácil acesso (E3).

Nas falas dos entrevistados, percebeu-se a relação da organização da Rede de Atenção à Saúde para quais as crianças são encaminhadas, com a melhora no desenvolvimento das crianças que utilizam serviços prestados pela rede: Ela já está inclusa em um serviço que é a escola, que já tem uma psicóloga, têm os professores que são especializados (AC2). A rede é assim, é o Centro de Referência de Assistência Social, a Unidade de Saúde, o Centro de Atenção Psicossocial, o Centro de Referência Especializado de Assistência Social, as escolas, os Centros Municipais de Educação Infantil, é tudo vinculado, então, quando tem alguma criança que precisa de alguma coisa, elas são diretamente vinculadas à rede (AC4). A atenção primária, o especialista e a reabilitação, quando as três coisas funcionam certinho, é quando tem o melhor resultado (M5).

\section{Discussão}

Os dados foram representados pela realidade de apenas seis Unidades de Saúde de um mesmo município, porém, para captar a adversidade de contexto, elegeram-se duas unidades de diferentes distritos, considerado limitações do estudo, não sendo possível, desta forma, generalizar os achados. Futuras pesquisas, com maior quantitativo amostral, são relevantes para dar seguimento aos resultados deste estudo.

Profissionais da Atenção Primária à Saúde relataram dificuldade para atendimento e o lidar com 
o cuidado das famílias de crianças com necessidades especiais. Os pontos-chave para o atendimento integral são as experiências que os profissionais de saúde recebem $^{(7)}$, inclusive no acolhimento, caracterizado como atendimento humanizado que possibilita o acesso das pessoas aos serviços ${ }^{(3)}$.

Nas falas dos profissionais que participaram desta pesquisa, foi referida a baixa procura das famílias em relação aos atendimentos na Unidade de Saúde. Ademais, foi citado que os serviços de saúde necessitam realizar a busca dessas crianças, o que pode ter relação com a literatura que demonstra baixa credibilidade das famílias para o atendimento das referidas crianças na Atenção Primária à Saúde ${ }^{(11)}$.

Enfatiza-se que o vínculo entre a equipe multiprofissional e a família deve se iniciar na primeira visita domiciliar ${ }^{(7)}$, após o nascimento da criança, devendo-se aproveitar dos momentos de acompanhamento para fortalecer a confiança na equipe e a relação de humanização do cuidado(3).

Os entrevistados reconheceram que as crianças com necessidades especiais têm os mesmos direitos que as demais, o que demonstra o conhecimento do conceito de igualdade. Entretanto, com necessidades diferentes, para que se tenham o direito de serem atendidas de forma integral, precisam de suporte diferenciado, seja por meio de capacitação dos profissionais para atendê-las, ambiência ou infraestrutura adequada. Os entrevistados revelaram que, entre a equipe, não há uniformidade no conhecimento sobre os direitos dessas crianças.

Em discordância com uma das falas, que retratou não existir prioridade de atendimento, existe normativa que estabelece a criação, a articulação e a ampliação dos pontos de atenção à saúde para pessoas com deficiência no Sistema Único de Saúde. A Rede de Cuidados à Pessoa com Deficiência garante a equidade na atenção à pessoa com deficiência ${ }^{(12)}$. Dentre as diretrizes, para o funcionamento dessa rede, destaca-se a promoção da equidade, a garantia de acesso de qualidade dos serviços de forma integral, com assistência multiprofissional e interdisciplinar, atenção humanizada e centrada nas necessidades das pessoas ${ }^{(13)}$.

A forma que as políticas públicas têm para melhorar o bem-estar e a integridade física das crianças é fornecendo o acesso às informações sobre direitos e aos programas de auxílio(14). Para que as famílias de crianças com necessidades especiais tenham conhecimento desses direitos, é necessário o suporte dos profissionais da saúde, para contribuir com a qualidade de vida dessas crianças e para que as políticas públicas sejam efetivamente implementadas ${ }^{(11)}$.

Os entrevistados demonstraram grande expectativa de que as famílias coordenem os cuidados das crianças. As famílias e os profissionais de saúde são responsáveis pela assistência e pelas orientações dos cuidados para melhorar a situação dessas crianças ${ }^{(7)}$. Os serviços de saúde devem permitir que as famílias compartilhem dificuldades e compreender adequadamente as fragilidades destas ${ }^{(5)}$. Várias etapas de cuidados são exigidas e reconhecidas como tensão e estresse emocional; diante disso, faz-se necessário que os profissionais de saúde compreendam e assegurem o apoio integral às famílias de crianças com necessidades especiais ${ }^{(15)}$.

Fundamentando as falas dos profissionais, referentes às orientações, é importante o diálogo entre a equipe e as famílias, com escuta qualificada, pois o suporte de informações pode reduzir o impacto negativo do diagnóstico de deficiência. Diante de várias falhas de estrutura e organização das Redes de Atenção à Saúde, as famílias ainda enfrentam dificuldades no acesso, na resolutividade dos serviços de saúde e na continuidade do tratamento das crianças, frequentemente sendo os fatores que desestimulam a procura de assistência na Atenção Primária à Saúde ${ }^{(11)}$.

Na organização do atendimento destinado às crianças com necessidades especiais, foram expressas, nas falas, limitações, como ausência de protocolos específicos para os atendimentos, pouco preparo da equipe e carência de capacitação. As falas indicaram, também, obstáculos, como a inadequação dos serviços 
existentes para atender às necessidades das crianças e respectivas famílias no território.

0 atendimento na Atenção Primária à Saúde apresenta perfil fragilizado, interferindo na qualidade da assistência, o que pode ser uma das causas da baixa inserção dessas crianças nos serviços de saúde ${ }^{(11)}$. Há despreparo para o atendimento das crianças com necessidades especiais diante da escassez de capacitação e formação dos profissionais sobre o assunto ${ }^{(4)}$. 0 cuidado direcionado às crianças também é de responsabilidade da Atenção Primária à Saúde, portanto, os profissionais dessas equipes necessitam estar aptos para garantir assistência de qualidade e resolutiva ${ }^{(11)}$.

Competeà gestão das Unidades deSaúde prestar apoio às equipes e realizar educação permanente em saúde, para oferecer assistência adequada às crianças com necessidades especiais ${ }^{(5)}$. A qualificação profissional em saúde deve estar em conformidade com as necessidades encontradas para assistência ${ }^{(16)}$.

A criação de trabalho digno aos profissionais de saúde da Atenção Primária à Saúde se torna eficaz no atendimento das necessidades de saúde das pessoas com deficiências, em contexto multidisciplinar $^{(16)}$. Com a oferta de capacitações, há garantia do acesso ao atendimento de saúde para todas as pessoas de maneira adequada, melhorando os resultados, considerando e respeitando os direitos e as necessidades da população( ${ }^{(3)}$.

Mesmo com a implementação de políticas de descentralização, ainda, é necessária medida mais eficaz e abrangente para alcançar cenário mais equitativo ${ }^{(17)}$. A ampliação e a qualificação da oferta de serviços no território e a coordenação do cuidado são atributos essenciais para assistência integral da Atenção Primária à Saúde ${ }^{(1)}$.

É necessário que os profissionais da Atenção Primária à Saúde tenham formação e qualificação, garantindo segurança na assistência. Considera-se relevante que os profissionais conheçam as políticas públicas que regulamentam as ações a serem procedidas $^{(3)}$, sendo importante, portanto, o incentivo de pro- fissionais qualificados para trabalhar nas Unidades de Saúde ${ }^{(18)}$.

A corresponsabilidade de outros profissionais pelo acompanhamento de crianças com necessidades especiais nas Redes de Atenção à Saúde, sendo uma ação intersetorial e interdisciplinar, com vistas a proporcionar qualidade do cuidado, apareceu nas falas dos entrevistados. Também emergiu que a atuação multiprofissional é imprescindível para investigação de solução de problemas de saúde. A reabilitação, para a pessoa com deficiência, gera promoção da saúde, pois a conquista de autonomia e a superação de limitações tendem a proporcionar melhor qualidade de vida para pessoa e família ${ }^{(3)}$.

Os profissionais da Atenção Primária à Saúde têm responsabilidade na detecção precoce dos sinais de alerta no desenvolvimento infantil das crianças com necessidades especiais, além de realizar o acompanhamento e executar o encaminhamento adequado das crianças para serviço especializado e de referência, quando necessário ${ }^{(2)}$.

As demandas de cuidados das crianças com necessidades especiais e das famílias são amplas para serem atendidas somente na Atenção Primária à Saúde. Expandir a coordenação do cuidado, para integrar os serviços e os programas de saúde a essas crianças, é crucial para o alcance de bons resultados. As crianças devem ter acesso à prevenção, à promoção, à terapêutica e à reabilitação ${ }^{(9)}$. A estratégia de matriciamento é fundamental, pois facilita esclarecimentos diagnósticos, bem como a organização das ações ${ }^{(19)}$.

Um modelo de trabalho coletivo, o trabalho em equipe intersetorial, estabelece articulação e influência mútua entre os profissionais, com saberes e práticas. A forma como os profissionais se envolvem e interagem influencia na rotina do trabalho. Com o propósito da atenção integral em saúde, a colaboração é fortalecida, com intuito de articular várias especialidades, para superação da fragmentação do trabalho e da assistência ${ }^{(3)}$.

Quando uma equipe ou um profissional de 
apoio matricial compartilha o conhecimento com a Estratégia Saúde da Família de referência, o apoio matricial auxilia a equipe na organização e execução de um projeto terapêutico para um sujeito, individual ou coletivo, que necessita de intervenção em saúde, sendo que a equipe de referência, também, pode apresentar dificuldades de realizar. A organização do sistema em Rede de Atenção à Saúde tem papel relevante e as equipes de Núcleo de Atenção à Saúde da Família são de extrema importância para garantia do direito integral à saúde das crianças com necessidades especiais e respectivas famílias ${ }^{(19)}$.

Os profissionais de saúde devem aconselhar e orientar as famílias sobre os encaminhamentos das crianças aos serviços da Rede de Atenção à Saúde, encorajando-as a partilhar quaisquer preocupações que possam ter e garantir a continuidade dos atendimentos na Atenção Primária à Saúde ${ }^{(20)}$.

Com objetivo de contribuir para visão ampla a respeito do objetivo de trabalho, é imprescindível que estejam alinhados os propósitos dos serviços de saúde, de maneira que todos se apropriem e utilizem de elementos que possibilitem uma equipe integrada, tornando a rede efetiva, pela construção da interprofissionalidade ${ }^{(2)}$.

\section{Conclusão}

Este estudo demonstrou que a organização da Atenção Primária à Saúde para atendimento às crianças com necessidades especiais e respectivas famílias, na perspectiva da equipe de saúde, expressa ambiguidade e despreparo dos serviços em relação aos direitos dessas crianças, na qualificação da equipe, bem como na exiguidade de protocolos específicos para normatizar tal atendimento. Considera-se que esses serviços, ao se organizarem e se articularem com a Rede de Atenção à Saúde para atender a essas famílias, podem garantir acesso aos serviços de saúde com qualidade e equidade, o que pode proporcionar melhoria nas condições de saúde dessas crianças.

\section{Colaborações}

Schuertz AL, Mazza VA, Seidel B e Ruthes VBTNM contribuíram na concepção, no desenho, na análise, na interpretação dos dados e na redação do manuscrito. Macedo LC colaborou com revisão crítica relevante do conteúdo intelectual e aprovação final da versão a ser publicada.

\section{Referências}

1. Santos AM, Almeida PF. Atención especializada en regiones de salud: desafios para garantizar el cuidado integral en Brasil. Rev Salud Pública. 2018; 20(3):301-7. doi: https://doi.org/10.15446/rsap. V20n3.61392

2. Condeles PC, BracarenseCF, Parreira BDM, Rezende MP, Chaves LDP, GoulartBF. Teamwork in the Family Health Strategy: professionals' perceptions. Esc Anna Nery. 2019; 23(4):e20190096. doi: https:// doi.org/10.1590/2177-9465-EAN-2019-0096

3. Almeida MHM, Pacheco S, Krebs S, Oliveira AM, Samelli A, Molini-Avejonas DR, et al. Avaliação da atenção primária em saúde por usuários com e sem deficiência. CoDAS 2017; 29(5):e20160225. doi:https://doi.org/10.1590/2317 1782/20172016225

4. Belmiro SSDR, Miranda FAN, Moura IBL, Carvalho SR, Monteiro AI. Practice of the primary health care nursing team towards children with disabilities. Rev Enferm UFPE On line. 2017. doi: 10.5205/ reuol.10438-93070-1-RV.1104sup201710

5. Iudici A, Favaretto G, Turchi GP. Community perspective: how volunteers, professionals, families and the general population construct disability: social, clinical and health implications. Dis Health J. 2018; 12(2):171-9. doi: https://doi. org/10.1016/j.dhjo.2018.11.014

6. Altman L, Zurynski Y, Breen C, Hoffmann T, Woolfenden S. A qualitative study of health care providers' perceptions and experiences of working together to care for children with medical complexity (CMC). BMC Health Serv Res. 2018; 18(1):70. doi: https://doi.org/10.1186/s12913018-2857-8 
7. Mattson G, Kuo DZ, Committee on Psychosocial Aspects of Child and Family Health and Council on Children with Disabilities. Psychosocial factors in children and youth with special health care needs and their families. Pediatrics. 2019; 143(1):e20183171. doi: https://doi. org/10.1542/peds.2018-3171

8. National Academies of Sciences, Engineering and Medicine. Opportunities for improving programs and services for children with disabilities. Washington, DC: The National Academies Press; 2018.

9. Dezoti AP, Alexandre AMC, Freire MHS, Mercês NNA, Mazza VA. Social support to the families of children with cerebral palsy. Acta Paul Enferm. 2015; 28(2):172-6. doi: https://doi. org/10.1590/1982-0194201500029

10. Bardin L. Análise de conteúdo. Lisboa: Edições 70; 2016.

11. Souza MHN, Nóbrega VM, Collet N. Social network of children with cronic disease: knowledge and practice of nursing. Rev Bras Enferm. 2020; 73(2):e20180371. doi: https://doi. org/10.1590/0034-7167-2018-0371

12. Machado WCA, Pereira JS, Schoeller SD, Júlio LC, Martins MMFPS, Figueiredo NMA. Comprehensiveness in the care network regarding the care of the disabled person. Texto Contexto Enferm. 2018; 27(3):e4480016. doi: http://dx.doi. org/10.1590/0104-07072018004480016

13. Dubow C, Garcia EL, Krug SBF. Percepções sobre a rede de cuidados à pessoa com deficiência em uma região de saúde. Saúde Debate. 2018; 42(117):455-67. doi: https://doi. org/10.1590/0103-1104201811709
14. Zeidan J, Shikako-Thomas K, Ehsan A, Maioni A, Elsabbagh M. Progress and gaps in Quebec's autism policy: a comprehensive review and thematic analysis. Can J Public Health. 2019; 1194):48596. doi: https://doi.org/10.17269/s41997-01900202-7

15. Bishop C, Small N, Parslow, Bowles D. Improving service coordination for children with complex needs. Br J Healthcare Manag. 2015; 21(10). doi: https://doi.org/10.12968/bjhc.2015.21.10.469

16. Declaration of Astana. Global Conference on Primary Health Care. Astana, Kazakhstan, 25-26 [Internet]. 2018 [cited Mar 13, 2020]. Available from:https://www.who.int/docs/default-source/ primary-health/declaration/gcphc-declaration

17. Eboreime EA, Eyles J, Nxumalo N, Eboreime OL, Ramaswamy R. Implementation process and quality of a primary health care system improvement initiative in a decentralized context: A retrospective appraisal using the quality implementation framework. Int J Health Plann Mgmt. 2019; 34:e369-e86. doi: https://doi. org/10.1002/hpm.2655

18. Wang X, Yang H, Duan Z, Pan J. Spatial accessibility of primary health care in China: a case study in Sichuan Province. Soc Sci Med. 2018; 209:14-24. doi: doi.org/10.1016/j.socscimed.2018.05.023

19. Santos RABG, Figueiredo LRU, Lima LC. Apoio matricial e ações na atenção primária: experiência de profissionais de ESF e NASF. Saúde Debate. 2017; 41(114):694-706. doi: https://doi. org/10.1590/0103-1104201711402

20. Choo YY, Agarwal P, How CH, Yeleswarapu SP. Developmental delay: identification and management at primary care level. Singapore Med J. 2019; 60(3):119-23. doi: https://doi.org/10.11622/ smedj.2019025 